\title{
Antimicrobial potential of some plant extracts against Candida species Isolated from infants oral thrush
}

\author{
Vian B. Nehmatullah ${ }^{1}$, Hero M. Ismael ${ }^{2}$, Dulair Chalabi ${ }^{1}$ and Shireen A. Amin ${ }^{2}$ \\ 1 Microbiology/ College of Medicine, Hawler Medical University \\ 2Biology/ College of Science Salahaddin University \\ Corresponding Author: Vian B. Nehmatullah, vianbadr@yahoo.com
}

\begin{abstract}
Background and objectives: Fungi infect billions of people every year, yet their contribution to the global burden of disease is mostly unrecognized. The study aimed to isolate and identify Candida spp. from oral infections in children, and evaluate the antifungal activity Fluconazole and Amphotericin B and Minimum Inhibitory Concentration (MIC) of plant extracts: thyme, pomegranate, and rosemary on three isolates of Candida including Candida albicans, $C$. parapsilosis and C. glabrata.
\end{abstract}

Methods: A total of 52 oral swab specimens were collected from children with signs and symptoms of oral thrush; 34 (65\%) were female, 18 (34.6\%) were male. The specimens were cultured on SDA medium then incubated for $24-48$ hrs at $37^{\circ} \mathrm{C}$.

Results: The results showed that $C$. albicans was the most frequently isolated species, which constitutes (63.6\%), followed by $C$. tropicalis, which was (18.2\%), followed by C. glabrata was $(9 \%)$. In comparison, the lowest rate of isolation were $C$. krusi and $C$. famata each were (4.6\%). The MIC of tested plant extracts (Pomegranate, Thyme, and Rosemary) and two antifungal agents (Fluconazole and Amphotericin B) against three most frequently isolated Candida spp., which were C. albicans, C. tropicalis, and C. glabrata were tested.

Conclusion: Pomegranate has the MIC against both $C$. albicans and $C$. glabrata at the fifth fold and has MIC against $C$. tropicalis at the third fold of dilutions, meanwhile thyme shows moderate inhibitory effects against both $C$. albicans and $C$. glabrata especially at the third folds, while rosemary have no any inhibitory effect against tested Candida species. Amphotericin B shows effective inhibitory effects against tested Candida especially $C$. glabrata at the fifth fold and $C$. albicans at the third fold, and $C$. tropicalis at the fourth fold of dilution. At the same time, fluconazole have only inhibitory effects against $C$. tropicalis and $C$. glabrata and have no any inhibitory effects against $C$. albicans.

Keywords: Candida spp., oral thrush, pomegranate, anti candida plant extract, Amphotericin B. 


\section{INTRODUCTION}

The Candida genus presents over 150 species, of which ten are responsible for infections in humans; Candida albicans is part of its normal microbiota in the oral cavity in human beings, a digestive, urinary, and reproductive tract of birds. ${ }^{1}$ and it is common to isolate few of these fungi from healthy animals. ${ }^{2}$ Oral Candidiasis is one of the common fungal infections affecting the oral mucosa. Candida albicans are one of the components of normal oral microflora, and around $30 \%$ to $50 \%$ of people carry this organism. ${ }^{3}$ There are many types of Candida species, which are seen in the oral cavity, Species of oral Candida are $C$. albicans, C. glabrata, C. guillermondii, C. krusei, C. parapsilosis, C. pseudotropicalis, $C$. stellatoidea, C. tropicalis. ${ }^{4}$

In clinical settings, Candidiasis is commonly treated with antimycotics-the antifungal drugs commonly used to treat Candidiasis are topical clotrimazole, topical nystatin, fluconazole, and topical ketoconazole. It is possible for $C$. albicans to develop a resistance to the drugs used to treat it, as seen from research done involving fluconazole, one of the medicines that are used to treat Candidiasis and noted about the emergence of fluconazole resistance. ${ }^{5}$ In this work, we have attempted to isolate and identify Candida species from infants infected with oral thrush and test the potential of antifungal activity of some selected plant extract against the most prevalent isolated Candida species.

\section{MATERIALS AND METHODS}

\section{Specimen collection and transportation}

The consent of the patients was made in writing, through the TIC (Term of Informed Consent) after this study is approved by the (Human Research Ethics Committee) of the University of Salahaddin/Erbil (No. 43/144 at / 1/8/2020) A total of 52 oral swab specimens were collected from children with signs and symptoms of oral thrush. The samples were safely transported to the laboratory under aseptic conditions for culturing.

\section{Culture incubation}

The swabs were inoculated on the surface of the sterilized slant SDA medium, and then they were incubated at $37^{\circ} \mathrm{C}$ (incubator Gallenkamp -9B/ England) $48 \mathrm{hrs}$; the negative cultures discard after one. ${ }^{6}$

\section{Yeast identifications}

Budding yeast cells of Candida spp. were identified microscopically. Candida species were differentiated from other yeasts by growing on (Yeast Malt Extract Agar) medium. ${ }^{6}$ The 
Yeast species have been identified by using API 20 AUX (Biomeriex- France) and for identification of Candida species API Candida (Biomeriex- France) was used.

\section{In vitro antifungal drug susceptibility testing}

The inoculum of three main isolated Candida spp. (C. albicans, C. tropicalis and C. glabrata) were prepared by suspending several colonies of grown cultures on Sabouraud's Dextrose Broth (SDB). Inoculum standardization was done using a standard procedure in which the fungal suspensions were adjusted spectrophotometrically to an absorbance of 0.6 at $530 \mathrm{~nm}$, which corresponds with the 0.5 McFarland standards. ${ }^{7}$

\section{Plant Extracts preparation}

The selected plant extracts were (Thyme, Pomegranate, and Rosemary). The plant samples used in the present study were collected from different locations. The plant samples were cleaned, washed by distilling water, then dried by placing it on filter paper in a shaded, set at room temperature, preserved in plastic sacs until used for extraction and chemical detections.

\section{Ethanol extractions of plants}

Twenty gram of each plant were weighted, then $200 \mathrm{ml}$ of ethanol (95\%) were added to it then mixed well in a Shaker (Shaker incubator-4046/Gallenkamp -9B/ England) for one hour and kept at $4 \mathrm{oC}$ for 24 hours, filtered through 4-5 layers of gauze and the supernatant were placed in Petri dish to dried out at room temperature, then the powder collected and preserved in vials in the refrigerator. ${ }^{8}$

\section{Antifungal agents preparation}

The selected plant extract (Thyme, Pomegranate, and Rosemary), in addition to two antifungal agents (Fluconazole and Amphotericin B), were tested against three main isolated Candida spp. including: ( $C$. albicans, $C$. tropicalis and $C$. glabrata), the inocula were prepared by suspending several colonies of grown cultures on Sabouraud's Dextrose Broth (SDB) according to the. ${ }^{9}$

\section{Disk diffusion method}

Each SDA plate was inoculated with the standard inoculum suspensions $(0.1 \mathrm{ml})$ by soaking a swab and rotating it over the agar plate. Six pores of diameter $(6 \mathrm{~mm})$ were made on each agar 


\section{Proceeding of the $2^{\text {nd }}$ Scientific Conference on Women's Health 2-3 September 2020 - Hawler Medical University}

plate with three replications for each. The pores were filled with appropriate antifungal suspensions. After 24 hours of incubation at $37^{\circ} \mathrm{C}$ zone of inhibition of growth was measured and recorded. ${ }^{9}$

\section{Minimum Inhibitory Concentration (MIC)}

The MIC was determined using the microdilution technique. Ninety-six-well microtiter plates with a U-shaped bottom were used. Initially, $100 \mu \mathrm{L}$ of Sabouraud Dextrose Broth (SDB) (Difco, India) was distributed in the plate holes. Then, $100 \mu \mathrm{L}$ of the prepared antifungal solution was transferred to the first well and serially diluted by transferring an aliquot of 100 $\mu \mathrm{L}$ from the most concentrated well to the next cavity. Thus, antifungal concentrations from 1-12 fold dilution were obtained. Finally, $100 \mu \mathrm{L}$ of inoculum corresponding to each strain was added to each cavity. ${ }^{10}$ The inocula were prepared in $0.9 \%$ saline, and the turbidity of the fungal suspensions was compared with that of the solution in tube 0.5 of the McFarland nephelometric scale, which corresponds to an inoculum of approximately $10^{6} \mathrm{CFU} / \mathrm{mL}$, and adjusted as needed. Then, the suspension was diluted in SDB to obtain an inoculum concentration of $10^{3} \mathrm{CFU} / \mathrm{mL}$. $^{11}$

Meanwhile, the viability of yeast strains (growth control) and medium sterility was controlled. Through serial dilution, from $1,000 \mu \mathrm{g} / \mathrm{mL}$ to $0.48 \mu \mathrm{g} / \mathrm{mL}$ were obtained. ${ }^{10}$

The test was performed in triplicate, and the microtiter plates were incubated at $37{ }^{\circ} \mathrm{C}$ for 24 h. A visual reading was performed to determine the MIC of each antifungal agent on Candida spp. strains. The formation of cell clumps ("buds") on the bottoms of the wells was considered. Thus, the lowest test product concentration that could visibly inhibit fungal growth was considered the MIC. ${ }^{11}$

Spectrophotometric readings were based on the reduction of growth compared to that in a growth control well for each isolate. For this, microtiter plates were agitated, and the optical densities of the wells were determined at $490 \mathrm{~nm} .{ }^{10}$

\section{RESULTS}

Figure 1 shows a pie chart of 52 specimens ware collected from children with oral thrush infections, 34 (65\%) were female, 18 (34.6\%) were male. 


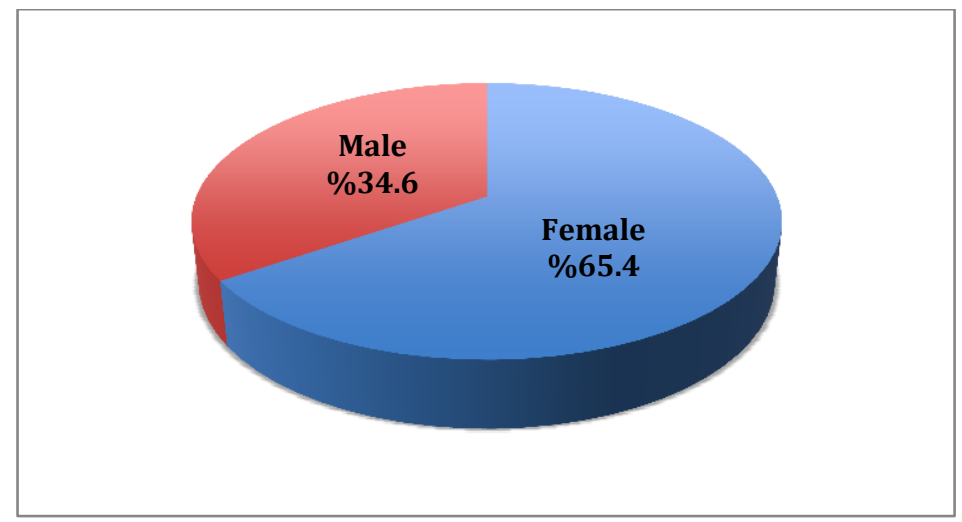

Fig 1: Pie chart shows the percentage of male and female infections

Figure 2 shows the percentage of Candida spp. isolated and identified in the present study, as shown in the result $C$. albicans was the most frequently isolated species, which constitutes (63.6\%), followed by C. tropicalis, which was (18.2\%), followed by C. glabrata was (9\%). In comparison, the lowest rate of isolation were C. krusi and C. famata each were (4.6\%).

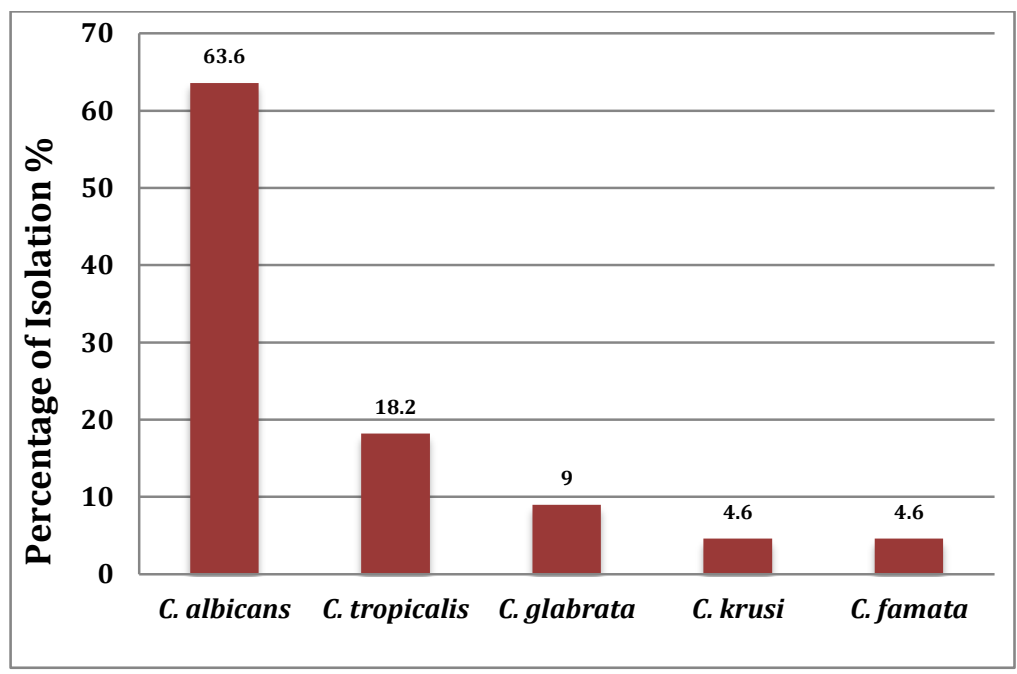

Fig 2: The percentage of Candida spp. infections in the present study

Figure 3 shows the means of inhibition diameter of tested plant extracts (Pomegranate, Thyme, and Rosemary) and two antifungal agents (Fluconazole and Amphotericin B) against three most frequently isolated Candida spp. which were C. albicans, C. tropicalis and $C$. glabrata. 


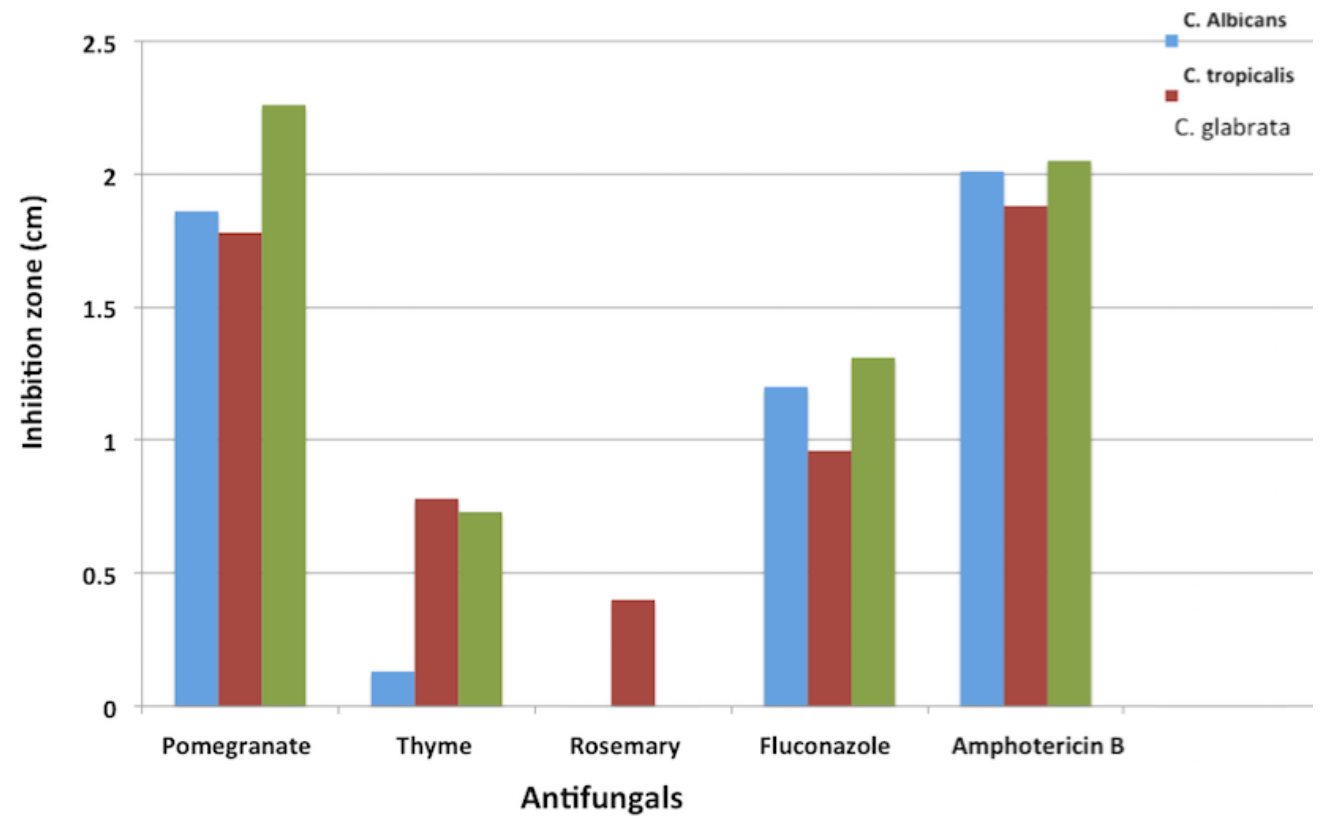

Figure3: Means of inhibition growth diameter obtained by disc diffusion method using plant extracts (Pomegranate, Thyme, and Rosemary) and two antifungal agents (Fluconazole and Amphotericin B) against three most frequently isolated Candida spp

Table 1 shows the MIC of tested plant extracts (Pomegranate, Thyme, and Rosemary) and two antifungal agents (Fluconazole and Amphotericin B) against the three most frequently isolated Candida spp., which were $C$. albicans, $C$. tropicalis and $C$. glabrata. As shown in the table, the pomegranate has the MIC against both $C$. albicans and C. glabrata at the fifth fold and has MIC against $C$. tropicalis at the third fold of dilutions, meanwhile thyme shows moderate inhibitory effects against both $C$. albicans and $C$. glabrata especially at the at third folds, while rosemary has no any inhibitory effect against tested Candida species.

The tested antifungal agents have different inhibitory effects against tested candida, Amphotericin B shows effective inhibitory effects against tested Candida especially $C$. glabrata at the fifth fold and C. albicans at third fold and C. tropicalis at the fourth fold of dilution, while fluconazole have only inhibitory effects against C. tropicalis and C. glabrata at sixth and fifth folds of dilution respectively, while having no any inhibitory effects against C. albicans. 
Table 1 : The MIC of tested plant extracts (Pomegranate, Thyme, and Rosemary) and two antifungal agents (Fluconazole and Amphotericin B) against three most frequently isolated Candida spp

\begin{tabular}{|c|c|c|c|c|c|c|c|c|c|c|c|c|c|c|}
\hline \multirow[t]{2}{*}{ Yeast } & & 1 & 2 & 3 & 4 & 5 & 6 & 7 & 8 & 9 & 10 & 11 & 12 & C \\
\hline & $\mathbf{P}$ & 0.921 & 0.576 & 0.392 & 0.389 & 0.311 & 0.383 & 0.531 & 0.580 & 0.589 & 0.592 & 0.589 & 0.608 & 0.62 \\
\hline \multirow{5}{*}{ 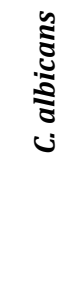 } & $\mathbf{T}$ & 0.443 & 0.451 & 0.435 & 0.531 & 0.537 & 0.519 & 0.500 & 0.523 & 0.492 & 0.574 & 0.570 & 0.580 & 0.62 \\
\hline & $\mathbf{R}$ & 0.558 & 0.465 & 0.452 & 0.399 & 0.424 & 0.405 & 0.406 & 0.416 & 0.437 & 0.420 & 0.426 & 0.426 & 0.61 \\
\hline & A & 0.546 & 0.424 & 0.382 & 0.393 & 0.438 & 0.448 & 0.466 & 0.462 & 0.461 & 0.460 & 0.463 & 0.473 & 0.61 \\
\hline & $\mathbf{F}$ & 1.596 & 1.035 & 0.729 & 0.544 & 0.566 & 0.578 & 0.578 & 0.588 & 0.582 & 0.582 & 0.572 & 0.603 & 0.61 \\
\hline & $\mathbf{P}$ & 0.485 & 0.470 & 0.378 & 0.449 & 0.475 & 0.440 & 0.455 & 0.450 & 0.431 & 0.495 & 0.489 & 0.486 & 0.51 \\
\hline \multirow{5}{*}{ 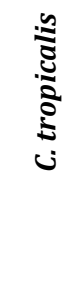 } & $\mathbf{T}$ & 0.422 & 0.440 & 0.376 & 0.426 & 0.457 & 0.405 & 0.526 & 0.586 & 0.581 & 0.641 & 0.683 & 0.652 & 0.52 \\
\hline & $\mathbf{R}$ & 0.744 & 0.664 & 0.690 & 0.678 & 0.648 & 0.657 & 0.462 & 0.620 & 0.557 & 0.693 & 0.587 & 0.592 & 0.5 \\
\hline & A & 0.498 & 0.485 & 0.393 & 0.388 & 0.423 & 0.439 & 0.446 & 0.498 & 0.488 & 0.506 & 0.550 & 0.443 & 0.52 \\
\hline & $\mathbf{F}$ & 1.619 & 0.878 & 0.669 & 0.602 & 0.649 & 0.518 & 0.629 & 0.658 & 0.659 & 0.659 & 0.639 & 0.639 & 0.51 \\
\hline & $\mathbf{P}$ & 1.082 & 0.796 & 0.526 & 0.588 & 0.473 & 0.465 & 0.543 & 0.524 & 0.468 & 0.497 & 0.545 & 0.522 & 0.649 \\
\hline \multirow{4}{*}{ 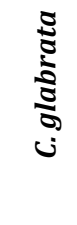 } & $\mathbf{T}$ & 0.666 & 0.589 & 0.530 & 0.530 & 0.539 & 0.569 & 0.552 & 0.506 & 0.559 & 0.585 & 0.474 & 0.616 & 0.642 \\
\hline & $\mathbf{R}$ & 1.049 & 0.668 & 0.562 & 0.544 & 0.584 & 0.634 & 0.577 & 0.599 & 0.593 & 0.620 & 0.563 & 0.652 & 0.649 \\
\hline & A & 0.813 & 0.606 & 0.472 & 0.466 & 0.434 & 0.573 & 0.545 & 0.576 & 0.618 & 0.465 & 0.473 & 0.516 & 0.643 \\
\hline & $\mathbf{F}$ & 1.696 & 1.135 & 0.688 & 0.605 & 0.483 & 0.564 & 0.613 & 0.504 & 0.509 & 0.617 & 0.576 & 0.551 & 0.644 \\
\hline
\end{tabular}

\section{DISCUSSION}

This results in a table (1) is partially in agreement with that found by ${ }^{12}$ who stated that nystatin and amphotericin B were the most drugs used locally for oral candidiasis treatment, fluconazole oral suspension is proving to be a very effective drug in the treatment of oral Candidiasis. Also agreement with that found by ${ }^{13}$ who screened for Fluconazole and its complex derivatives antifungal activity against $C$. albican and Asp. niger by MIC method. And they stated that the complexes showed varied activity ranging from 2-20\%.

The results showing significant sources of quinic acid, gallic acid, and rutin in the root, which might give an original source of this biological antifungal activity and antioxidant. ${ }^{14}$

The result is in agreement with that found by ${ }^{15}$ who detected their Minimum Inhibitory Concentration (MIC) against Candida spp isolated from dental floss; they discovered that Terbinafine was the most effective antifungal drug against $C$ albicans and $C$. glabrata with (MIC) range $(0.149$ and $0.075 \mu \mathrm{g} / \mathrm{ml})$ 


\section{CONCLUSIONS}

Candida albicans was the most frequently isolated yeast from oral thrush infections $63.6 \%$, pomegranate was the most effective plant extract with MIC 3.18 specially against $C$. albicans and $C$. tropicalis,. The antifungal drug Amphotericin B posses the highest MIC against $C$ albicans and C. glabrata.

\section{Conflict of interests:}

The author declares no competing interests.

\section{REFERENCES}

1. Mancianti F., Nardoni S. \& Ceccherelli R.. Occurrence of yeasts in psittacines droppings from captive birds in Italy. Mycopathologia 2002.153:121-124.

2. Tekeli A, Dolapci, I, Emral R and Cesur S. Candida carriage and Candida dubliniensis in oropharyngeal samples of type-1 diabetes mellitus patients. Mycoses.2004. 47(7): 315318.

3. Dangi YS, Soni MS, Namdeo KP. Oral Candidiasis: A review. Int J Pharm Pharm Sci. 2010. 2: 36-41.

4. Terezhalmy GT and Huber MA. Oropharyngeal Candidiasis: Etiology, epidemiology, clinical manifestations, diagnosis, and treatment. Crest Oral-B at dentalcare.com Contin Educ Course.2011:1-16.

5. Udhaya V. Role of Candida in Cervical Cancer - Association and Characterization of Candida, immuno diagnosis of Vaginal/cervical Candidiasis and anticandidal activity of certain medicinal plants. 2001. J. Infect. Dis. 5:4.2-5.

6. Mc Ginnes. Laboratory Hand Book of Medical Mycology. Academic Press.1980. 661 pp.

7. Siddiqui ZN, Farooq F, Musthafa TNM, Ahmad A, Khan AU. .Synthesis, characterization, and antimicrobial evaluation of novel halopyrazole derivatives. J Saudi Chem Soc. 2013 .17: 237- 243.

8. Grand, A.; Verpoort, R.; Wondergem, P. A. and Ponsset, J. L.. Anti infection phytotherapies of the tree-savannah sengal (west-Africa), 11- antimicrobial activity of 33 species. J. Ethnoph. 1988. 22:25-31. 
Proceeding of the $2^{\text {nd }}$ Scientific Conference on Women's Health 2-3 September 2020 - Hawler Medical University

9. Ismael, H. M.. Prevalence of dermatophytes and non-dermatophytic fungi in a rural village of Iraqi Kurdistan with special reference to their inhibition by some natural plant extract. Ph.D. Thesis. 2009 College of Science. Salahaddin- Erbil Univ. Iraq

10. de Castro, R. D., Pereira, Trícia M., de Souza, A., Bezerra, Louise M. D., Ferreira, G. LS., de Brito C., Edja, M. M. and Cavalcanti, A. L. Antifungal activity and mode of action of thymol and its synergism with nystatin against Candida species involved with infections in the oral cavity: an in vitro study. BMC Complementary and Alternative Medicine .2015. 15:417

11. Clinical and Laboratory Standards Institute (CLSI). Protocol M27-A2. Reference method for broth dilution antifungal susceptibility testing of yeasts. 2nd ed. Pennsylvania: NCCLS; 2002.

12. Garcia-Cuesta C, Sarrion-Pérez MG and Bagán JV. .Current treatment of oral candidiasis: A literature review. J Clin Exp Dent. 2014. 6(5): e576-e582.

13. Ali M., Ahmed M, Ahmed S., Syed Imran Ali, Samina Perveen, Majid Mumtaz, Syed Moazzam Haider, and Urooj Nazim .Fluconazole and its interaction with metal (II) complexes: SEM, Spectroscopic and antifungal studies, Pak. J. Pharm. Sci., 2017. 30(1): 187-194

14. Ismael BQ, Alma MH, Ismael HM, GoranYA, Galalaey AM, Göçeri A, Ahmad SS, and Khalid H.. Phytochemical Profile and Antifungal Effect of (Quercus infectoria Oliv.) Plant Root Extract on Several Candida spices. International Journal of Scientific \& Engineering Research. 2018.9 (3):600-612.

15. Ismael HM, Nehmatullah VB, Al-Barzanji HA, and Hussain SK. Minimum inhibitory concentration (MIC) determination of four antifungal agents against Candida spp. isolated from patients in Erbil city, The $5^{\text {th }}$ International Conference on Applied Science, Energy and Environment ICASEE April 2018. 